\title{
Relación entre autoconcepto y ansiedad estado-rasgo en universitarios peruanos
}

\author{
The relationship between self-concept and state-trait anxiety in peruvian \\ university students
}

\author{
Claudia Karina Guevara-Cordero ${ }^{1}$ \\ Universidad Privada del Norte \\ Nikolai Martin Rodas-Vera ${ }^{2}$ y Roxana Patricia Varas-Loli ${ }^{3}$ \\ Universidad César Vallejo, Lima, Perú
}

Recibido: $24-11-19$

Aceptado: $17-12-19$

\section{Resumen}

La ansiedad es un problema de salud pública que debe ser investigado. Teóricamente, el autoconcepto está relacionado con la ansiedad. Por este motivo, el presente estudio se planteó el objetivo de encontrar la relación entre estas dos variables. Para ello, se diseñó una investigación no experimental, transversal y correlacional simple, en la cual se utilizó la Escala de Ansiedad Estado-Rasgo y el Cuestionario de Autoconcepto de Garley, el cual fue adaptado para universitarios. La muestra estuvo conformada por 150 jóvenes universitarios de Lima, entre los 17 y 27 años de edad, de ambos sexos. Los resultados indican relaciones significativas y con tamaños del efecto que oscilan entre bajo, moderado y grande entre ansiedad rasgo y las dimensiones de autoconcepto (físico, social, familiar, intelectual, personal y control), pero la ansiedad estado solo se relacionó de manera significativa con autoconcepto intelectual, personal y control. Finalmente, se discuten los hallazgos en función de los antecedentes, las bases teóricas y las limitaciones, elaborando recomendaciones para futuras investigaciones.

Palabras clave: Ansiedad estado-rasgo; autoconcepto; estudio correlacional; universitarios.

\begin{abstract}
Anxiety is a public health problem that must be researched. Theoretically, self-concept is related to anxiety. Because of this, the aim of this study is to find the relationship between these two variables. In order to achieve it, a simple non-experimental, transversal and correlational research was designed. In this investigation, the State-Trait Anxiety State Scale and an adapted version of the Garley Self-concept Questionnaire were used. The sample
\end{abstract}

\footnotetext{
1 Docente, Universidad Privada del Norte. E-mail: claudia.guevara@upn.edu.pe, claudia.guevara.cordero@gmail.com 2 Docente, Universidad César Vallejo. E-mail: nikolairodas@gmail.com,nrodas@ucv.edu.pe 3 Docente, Universidad César Vallejo. E-mail: rvaras@ucv.edu.pe

(C) Los autores. Este artículo es publicado por la Revista de Investigación en Psicología de la Facultad de Psicología, Universidad Nacional Mayor de San Marcos. Este es un artículo de acceso abierto, distribuido bajo los términos de la licencia Creative Commons Atribucion - No Comercia_Compartir Igual 4.0 Internacional. (http://creativecommons.org/licenses/by-nc-sa/4.0/) que permite el uso no comercial, distribución y reproducción en cualquier medio, siempre que la obra original sea debidamente citada.
} 
consisted of 150 young university students from Lima, between the ages of 17 and 27, of both sexes. The results indicated significant relationship and different levels of the effect size from low, moderate and large between trait anxiety and the dimensions of self-concept (physical, social, familiar, intellectual, personal and control), but state anxiety was only significantly related to intellectual, personal and control dimensions of self-concept. Finally, the findings are discussed based on the background information, the theoretical basis and limitations, developing recommendations for future research.

Keywords: Trait-state anxiety; self-concept; correlational study; university students.

La ansiedad es un problema de salud mental que afecta a niños (Sandín, Chorot, Santed, \& Valiente, 2002), adolescentes (Ospina-Ospina, Hinestrosa-Upegui, Paredes, Guzmán, \& Granados, 2011), adultos mayores (Quiroz, \& Flores, 2007), entre otras poblaciones. En universitarios, se revela que la ansiedad puede presentarse en niveles bajos (Vélez, Garzón, \& Ortíz, 2008; González, Landero \& García-Campayo, 2009), pero también puede existir prevalencia de niveles altos (Dominguez-Lara, Bonifacio-Vilela \& Caro-Salazar, 2016; Cardona-Arias, PerezRestrepo, Rivera-Ocampo, Gómez-Martínez \& Reyes, 2015).

Por este motivo, es necesario investigar las variables que pueden hacer frente a la ansiedad. Así, se ha encontrado que a mayor procrastinación académica, mayor ansiedad-rasgo (Bolívar, Ballesteros \& Ramírez, 2014), que la comprensión emocional y la regulación emocional pueden ser factores que se relacionan de manera inversa con la ansiedad estado y rasgo (Rodríguez, Amaya \& Argota, 2011), que mientras más crece la autoeficacia percibida (García \& Velázquez, 2018), y a mayor solución de problemas como estrategia de afrontamiento (Cárdenas, Castillo \& Camargo, 2011) menos ansiedad se experimenta.

Una variable que puede ser relevante en la protección de la ansiedad en universitarios es el autoconcepto, dado que tanto en adolescentes españoles (Caballo et al. 2018; Núñez \& Crismán, 2016) como en universitarios peruanos (Rodrich, 2019), la autoestima, que es una variable muchas veces concebida como similar al autoconcepto, se relaciona de manera inversa con diferentes formas de ansiedad.

Además, en adolescentes españoles, el autoconcepto académico y familiar han mostrado relación inversa con diversas variables como la agresión, la visión negativa del mundo y el consumo de drogas; el autoconcepto social, emocional y físico se han relacionado inversamente con la visión negativa del mundo y la competencia social; todas las dimensiones del autoconcepto han mostrado relación inversa con la inestabilidad emocional (Fuentes, García, Gracia, \& Lila, 2011). 
Asimismo, las dimensiones de autoconcepto personal y físico evidencian relación significativa e inversa con ansiedad en adolescentes peruanos y adultos españoles (Alvarado, 2015; Sánchez, López, Moral \& Zagalaz, 2017); en niños peruanos las dimensiones personal, intelectual y control del autoconcepto se relacionan de manera directa con el humor, la empatía y la creatividad de la resiliencia (Matalinares et al 2011); en tanto que en población universitaria española, las dimensiones de autoconcepto tienen una relación inversa con ansiedad estado y rasgo (Alonso, Lucas, Lobera, \& Izquierdo, 2006).

Una de las teorías más aceptadas para definir, explicar y medir el autoconcepto (Esnaola, Goñi, \& Madariaga, 2008) es la de Shavelson, Hubner, y Stanton (1976), que lo definieron como la percepción y evaluación que una persona tiene de sí misma. Así, este presenta siete características: es organizado, multidimensional, jerárquico, estable, evolutivo, evaluativo y diferenciable.

Es organizado porque existen categorías, como familia, escuela, amigos, que dan significando a las experiencias. De esta manera, estas categorías generan que sea multidimensional. Es jerárquico, porque en la cima se encuentra el autoconcepto general, el cual puede dividirse en autoconcepto académico y autoconcepto no académico, del cual puede derivarse el autoconcepto social, emocional y físico. Es estable, a pesar de que en situaciones específicas la percepción de uno mismo podría cambiar. Es evolutivo, porque se forma mientras el niño crece, aprende a diferenciarse del ambiente y va adquiriendo las categorías mencionadas. Es evaluativo, porque cada persona otorga determinada importancia a cada dimensión del autoconcepto. Finalmente, es diferenciable de otros constructos.

Estos autores conciben de igual manera a la autoestima y al autoconcepto, pero si bien ambos constructos tienen una marcada influencia social (Massenzana, 2017), la autoestima probablemente se refiere al resultado de la autoevaluación, a la satisfacción personal producto del autoconcepto (García \& Musitu, 2014).

Acerca del instrumento empleado, se prefirió el Cuestionario de Autoconcepto de Garley (CAG, García, 2001) y no el Cuestionario de Autoconcepto AF5 (García \& Musitu, 2001, 2014), porque si bien la estructura teórica de este último ha sido confirmado en algunos estudios (Londoño, Lubert, Sepúlveda, \& Ferreras, 2019), se ha encontrado que con técnicas estadísticas robustas, la dimensión física se divide en dos, configurando un modelo teórico de seis dimensiones (Esnaola, Rodríguez \& Goñi, 2011). Sin embargo, el criterio principal fue que el CAG presenta una dimensión importante, el autoconcepto de control, que está teóricamente relacionada a la posibilidad del manejo de la ansiedad.

Así, las dimensiones del CAG son: autoconcepto físico, relacionado a la aceptación del propio aspecto físico; autoconcepto social, evaluación de la habilidad para relacionarse con los demás y ser aceptado por los amigos; autoconcepto 
intelectual, evaluación de las capacidades intelectuales; autoconcepto familiar, evaluación del vínculo y la satisfacción familiar; sensación de control, valoración de la medida en que el sujeto se considera como autor de sus comportamientos y de las situaciones; y el autoconcepto personal, como una evaluación general como persona.

Por otra parte, muchos teóricos han estudiado la ansiedad y la han considerado como una emoción, un rasgo de personalidad o un desorden clínico (Eysenck, 2014), brindando diversas explicaciones, entre las que se encuentran factores genéticos, emocionales, familiares, cognitivos, de aprendizaje, entre otros (Emmelkamp, \& Ehring, 2014), para una variadas clases de ansiedad, como ansiedad ante los exámenes, ansiedad social y fobias.

Los tipos de ansiedad estudiados en este trabajo, son la ansiedad rasgo (AR) y estado (AE). La AR es una disposición comportamental o tendencia latente a percibir las situaciones inofensivas como amenazadoras, lo que conllevaría a aumentar desproporcionadamente la $\mathrm{AE}$, que estaría caracterizada por la fuerza con la que aparecen las reacciones ansiosas, como tensión, aprehensión o agitación del sistema nervioso autónomo, las cuales son transitorias (Spielberger, 1966; Spielberger, Gorsuch \& Lushene, 2015).

Respecto a la AE, estas respuestas ansiosas son interpretadas como una señal que inician una secuencia de procesos cognitivos o motores destinados a evitar o a afrontar la situación amenazante; en cambio, la AR refleja las experiencia pasada que influye en determinar algunos estímulos como peligrosos (Spielberger, 1966). Según lo planteado por Spielberger et al (2015), las personas con más ansiedad rasgo "son más propensos a responder con un aumento de $\mathrm{AE}$ en situaciones de relaciones interpersonales que impliquen una amenaza a la autoestima" (p. 10). Por este motivo

En base a los antecedentes y al marco teórico, la presente investigación se planteó como objetivo encontrar la relación que existe entre las dimensiones de autoconcepto y las subescalas de ansiedad, en una muestra de universitarios, hipotetizando que existen relaciones inversas, moderadas y significativas.

\section{MÉTODO}

\section{Diseño}

Se realizó una investigación empírica, empleando la estrategia asociativa (Light, Singer \& Willett, 1990, Arnau, 1995), en su diseño correlacional simple, donde se examinó la relación entre las dimensiones de las dos variables estudiadas, recogiendo la información de cada participante una sola vez (transversal) y sin controlar estadísticamente otras variables. 


\section{Procedimiento}

En primer lugar se realizó la adaptación del CAG en base a las directrices de la Comisión Internacional del Test (Muñiz, Elosua, \& Hambleton, 2013). Para tal fin, se analizó la existencia del constructo en la población diana, concluyéndose que los universitarios también pueden presentar las mismas dimensiones que los escolares; esta conclusión se basó en antecedentes donde se ha probado el constructo en universitarios (e.g. Bustos, Oliver, \& Galiana, 2015; López, Esteban, Peris, Ros, $\&$ Carbonell, 2009). Cada cambio fue realizado conservando el significado de los ítems y evaluando la comprensión de los mismos por los participantes, lo cual fue evaluado mediante un estudio piloto, donde los sujetos fueron representativos de la población.

Los instrumentos fueron recogidos en una universidad nacional de Lima y los aplicadores fueron estudiantes de psicología, quienes estuvieron familiarizados con la cultura de la universidad y fueron capacitados en rapport, asentimiento informado y uso del instrumento (Muñiz et al 2013). Finalmente, los datos fueron procesados en SPSS y AMOS 25 (Arbuckle, 2017).

\section{Análisis de datos}

En principio, se evaluó las propiedades psicométricas de los instrumentos, ya que el Cuestionario de Autoconcepto de Garley fue adaptado para el presente estudio con muestra de universitarios. Para el análisis de la estructura interna se empleó análisis factorial confirmatorio, donde se considera como favorable valores de $\mathrm{x}^{2} /$ $\mathrm{gl}<2$ e índice de ajuste comparativo (CFI) $\geq .90$ (Bentler, 1990), mientras que en raíz residual estandarizada cuadrática media (SRMR) y el error cuadrático medio de aproximación (RMSEA) se consideran aceptable valores $\leq .08$ (MacCallum, Browne \& Sugawara, 1996).

Para evaluar la relación entre las variables se empleó el Rango Spearman de correlación de orden $\left(\mathrm{r}_{\mathrm{S}}\right)$ y el coeficiente de determinación $\left(\mathrm{r}^{2}\right)$ para el tamaño del efecto, donde $r=.10$ (tamaño del efecto pequeño), $r=.30$ (tamaño del efecto mediano) y $\mathrm{r}=.50$ (tamaño del efecto grande) (Cohen, 1988).

\section{Muestra}

Se utilizó un muestreo no probabilístico (Hernández, Fernández \& Baptista, 2014) e intencional (Sánchez \& Reyes, 2015). La muestra fue evaluada en el interior de una universidad nacional, previo asentimiento de los participantes, que llegaron a 150, de los cuales 81 fueron varones y 69 mujeres. Las edades oscilaron entre los 17 y 27 años. Las carreras profesionales a las que pertenecían fueron Letras, Ciencias Sociales, Ciencias Contables, Ciencias de la Salud y de diversas áreas de Ingeniería. Cada participante brindó su autorización para participar en la investigación, firmando un consentimiento informado, en la que se explicada 
los objetivos y la posibilidad de renunciar la encuesta, entre otros aspectos de integridad académica.

\section{Instrumentos}

Cuestionario de Autoconcepto de Garley. Para la presente investigación se realizó una adaptación del Cuestionario de Autoconcepto de Garley (García, 2001) a la muestra usada. Se encontró evidencia de validez por estructura interna empleando análisis factorial confirmatorio y evaluando un modelo no jerárquico oblicuo. Los índices de ajuste hallados se encontraron en los límites aceptados (SRMR=.06, $\mathrm{X}^{2}$ / $\mathrm{gl}=1.3, \mathrm{CFI}=.95, \mathrm{RMSEA}=.05$ ). La confiabilidad de las puntuaciones fue analizada por el método de consistencia interna, mediante los coeficientes alfa y omega compuesto. Autoconcepto físico obtuvo $\mathrm{Alfa}=.83$ y omega $=.84$, quedado con 3 ítems; autoconcepto social obtuvo alfa $=.73$ y omega $=.73$, con 3 ítems; familiar alcanzó alfa $=.66$ y omega $=.68$, con 3 ítems; intelectual obtuvo alfa $=.70$ y omega $=$ .70 , con 3 ítems; autoconcepto personal obtuvo alfa $=.84$ y omega $=.85$, quedando con 3 ítems; y control obtuvo alfa $=.63$ y omega $=.63$, con 4 ítems. Las dimensiones familiar y control obtuvieron coeficientes por debajo del valor de aceptación, por lo que las interpretaciones deben ser consideradas como una aproximación. Se optó por analizar la confiabilidad de cada dimensión debido a que no fue probado un modelo de segundo orden ni bifactor (Dominguez-Lara, \& Rodriguez, 2017; Flores-Kanter, Dominguez-Lara, Trógolo \& Medrano, 2018). Producto de la adaptación y el análisis psicométrico, el test finalmente quedó con 19 ítems, con cinco opciones de respuesta, que van desde nunca hasta siempre.

Escala de Ansiedad Estado-Rasgo. Se empleó la Escala de Ansiedad EstadoRasgo (STAI, Spielberger, Gorsuch \& Lushene, 2008; adaptación española, Buela-Casal, Guillén-Riquelme, Seisdedos, 2015) y se evaluaron sus propiedades psicométricas. La evidencia de validez examinada fue la de estructura interna por medio de análisis factorial confirmatorio, donde se puso a prueba un modelo no jerárquico oblicuo. Los índices de ajuste se encontraron en los límites aceptados $\left(\mathrm{SRMR}=.04, \mathrm{X}^{2} / \mathrm{gl}=1.2, \mathrm{CFI}=.99, \mathrm{RMSEA}=.04\right)$. El método de confiabilidad fue el de consistencia interna, calculado mediante el coeficiente alfa y el omega compuesto. Ansiedad estado obtuvo alfa $=.71$ y omega $=.78$, con 20 ítems; y ansiedad rasgo obtuvo alfa $=.89$ y omega $=.70$, con 20 ítems. También se optó por analizar la confiabilidad de cada factor debido a que no fue probado un modelo de segundo orden ni bifactor (Dominguez-Lara, \& Rodriguez, 2017; Flores-Kanter, et al. 2018). Las cuatro opciones de respuesta de ansiedad estado van desde nada a mucho, mientras que las cuatro de ansiedad rasgo, desde casi nunca hasta casi siempre.

\section{RESULTADOS}

Como se puede apreciar en la Tabla 1, la ansiedad estado correlaciona de manera inversa con todas las dimensiones de autoconcepto, pero la relación es pequeña 
y no significativa con las dimensiones de autoconcepto físico, social y familiar; mientras que es significativa con las demás. Se puede observar que el tamaño del efecto entre ansiedad estado y autoconcepto personal es moderado, mientras que con autoconcepto intelectual y control es pequeño.

\section{Tabla 1}

Correlación entre ansiedad estado y las dimensiones de autoconcepto

\begin{tabular}{lcccccccc}
\hline & & AFi & AS & AFa & AI & AP & AC \\
\hline Ansiedad estado & $\mathrm{r}_{\mathrm{S}}$ & -.05 & -.07 & -.09 & -.25 & -.39 & -.28 \\
& $\mathrm{p}$ & .553 & .431 & .301 & .002 & .001 & .001 \\
& $\mathrm{r}^{2}$ & -- & -- & -- & 0.06 & $\mathbf{0 . 1 5}$ & 0.08 \\
\hline
\end{tabular}

Nota. $\mathrm{r}_{\mathrm{S}}$ : Rango Spearman de correlación de orden, $p$ : Nivel de significación, $\mathrm{r}^{2}$ :Coeficiente de determinación, AFi: Autoconcepto físico, AS: Autoconcepto social, AFa: Autoconcepto familiar, AI: Autoconcepto intelectual, AP: Autoconcepto personal, AC: Control. En negrita: Tamaños del efecto moderados.

En la Tabla 2 se observa que la ansiedad rasgo tiene una relación inversa y significativa con todas las dimensiones de autoconcepto. Con la dimensiones autoconcepto físico y social, la relación es pequeña; mientras que con las dimensiones de autoconcepto familiar, intelectual y control el tamaño del efecto es moderado. Sin embargo, con el autoconcepto personal, la relación conlleva a un tamaño del efecto grande.

Tabla 2

Correlación entre ansiedad rasgo y las dimensiones de autoconcepto

\begin{tabular}{llcccccc}
\hline & & $\mathbf{A F i}$ & $\mathbf{A S}$ & $\mathbf{A F a}$ & $\mathbf{A I}$ & $\mathbf{A P}$ & $\mathbf{A C}$ \\
\hline Ansiedad rasgo & $\mathrm{r}_{\mathrm{S}}$ & -.24 & -.19 & -.33 & -.35 & -.50 & -.47 \\
& $\mathrm{p}$ & .003 & .021 & .001 & .001 & .001 & .001 \\
& $\mathrm{r}^{2}$ & .06 & .04 & $\mathbf{. 1 1}$ & $\mathbf{. 1 3}$ & $\mathbf{. 2 5}$ & $\mathbf{. 2 2}$ \\
\hline
\end{tabular}

Nota. $r_{\mathrm{S}}$ : Rango Spearman de correlación de orden, $p$ : Nivel de significación, $\mathrm{r}^{2}$ : Coeficiente de determinación, AFi: Autoconcepto físico, AS: Autoconcepto social, AFa: Autoconcepto familiar, AI: Autoconcepto intelectual, AP: Autoconcepto personal, AC: Control. En negrita: Tamaños del efecto moderados. En negrita y cursiva: Tamaño del efecto grande.

\section{DISCUSIÓN}

Debido a que la ansiedad es un problema de salud mental que muestra una significativa prevalencia en universitarios, el objetivo de la presente investigación consistió en aportar evidencias de correlación con una variable que teóricamente está presente en su desarrollo, como es el autoconcepto (Spielberger et al, 2015).

Los resultados indican que la ansiedad rasgo está relacionada de manera significativa con todas las dimensiones de autoconcepto (físico, social, familiar, intelectual, personal y control); en cambio, la ansiedad estado solo se relacionó de manera significativa con las dimensiones de autoconcepto intelectual, personal y control. 
En las investigaciones estudiadas, se ha encontrado esta tendencia. No obstante, Alonso et al (2006), había encontrado que la relación inversa significativa se produce entre ambas subescalas de ansiedad y todas las dimensiones de autoconcepto; aunque, esto pudo haberse afectado porque el instrumento de autoconcepto empleado es diferente.

Sánchez et al (2017) coincidieron en que la ansiedad rasgo se relaciona de manera inversa con el autoconcepto físico; sin embargo, también encontraron que la ansiedad estado se relaciona de esta manera con el autoconcepto físico, lo cual no fue corroborado por el presente estudio. Esto se puede deber a que en aquella investigación, la población fueron usuarios de gimnasios. En la población de universitarios de este estudio, sí se halló una relación significativa e inversa entre autoconcepto intelectual y ansiedad estado y rasgo, lo cual muestra que en universitarios la valoración que se tiene de uno mismo en el aspecto académico es importante para que las experiencias o situaciones que se afronten sean percibidas menos amenazantes y se presenten menos reacciones ansiosas que perturben.

En el presente estudio, un aspecto que llama la atención es la relación con tamaño del efecto moderado entre la ansiedad estado y el autoconcepto personal, lo que significa que si un universitario tiene una apreciación global adecuada de sí mismo, podría estar mejor preparado para tener menos reacciones ansiosas, menos tensión o aprehensión. Esta misma orientación se produce con la ansiedad rasgo, aunque aquí, el tamaño del efecto es grande, probablemente porque ambas variables se estarían comportando como rasgos estrechamente relacionadas con la personalidad y el aprendizaje (Eysenck, 2014; Emmelkamp \& Ehring, 2014).

Se ha encontrado también que el autoconcepto familiar y social está relacionado significativamente con la ansiedad rasgo; esto apoya la idea de que la valoración de los sistemas de socialización integrados en la percepción de uno mismo (Nunes, Rigotto, Ferrari \& Marín, 2012), se podrían agregar a los factores protectores frente a la ansiedad como rasgo.

Se había adaptado el CAG a universitarios debido a que presenta una dimensión de control en el autoconcepto, y se había hipotetizado una relación inversa entre este y las subescalas de ansiedad, lo cual fue confirmado. Esto significa que mientras una persona se siente capaz de controlar lo que le pasa, menos ansioso será y se mostrará. Estos hallazgos van acorde a la investigación sobre ansiedad y regulación emocional (Rodríguez et al 2011) y autoeficacia (García \& Velázquez, 2018).

Existen limitaciones que deben tenerse en cuenta al momento de generalizar los resultados. Así, el muestreo no siguió las pautas de la probabilidad, por lo que los datos expuestos deben aplicarse a universitarios con las mismas características presentadas. Así mismo, la confiabilidad de autoconcepto familiar y control, 
resultaron menos de lo aceptado, por lo que la estabilidad de las puntuaciones en estas dimensiones puede estar dependiendo de factores aleatorios.

Futuras investigaciones probando psicométricamente este instrumento adaptado en universitarios, permitirán conocer mejor su estructura. Asimismo, en posteriores investigaciones se deberán controlar estadísticamente variables externas y se deberá ampliar la cantidad de participantes. También debido al estado del arte de estas dos variables, se las podría incluir en modelos de ecuaciones estructurales. Sin embargo, se puede concluir que en universitarios, entenderse bien y estar satisfecho consigo mismo está asociado a tener menos ansiedad rasgo y estado.

\section{CONCLUSIONES}

1. En la muestra de universitarios de Lima, se encontró una relación inversa y significativa entre ansiedad estado y autoconcepto intelectual, personal y control, donde se evidencia tamaño del efecto pequeño entre ansiedad estado y autoconcepto intelectual y control; mientras que el tamaño del efecto es moderado entre ansiedad estado y autoconcepto personal.

2. La relación entre ansiedad estado y autoconcepto físico, social y familiar resultó no significativa.

3. Existe una relación inversa y significativa entre ansiedad rasgo y todas las dimensiones de autoconcepto. El tamaño del efecto fue grande entre ansiedad rasgo y autoconcepto personal; moderado entre ansiedad rasgo y autoconcepto familiar, intelectual y control; pequeño entre ansiedad rasgo y autoconcepto físico y social.

\section{REFERENCIAS}

Alonso, J. L. N., Lucas, J. M. A., Lobera, F. G., \& Izquierdo, J. G. N. (2006). Relación entre autoconcepto y ansiedad en estudiantes universitarios. International Journal of Developmental and Educational Psychology, 1(1), 243-245. Disponible en: https:// www.redalyc.org/pdf/3498/349832311021.pdf

Alvarado, H.A. (2015). Autoconcepto personal y nivel de ansiedad en estudiantes del VII ciclo de educación secundaria de la Institución Educativa "San Martín de Porres" del Distrito de Ate, 2014. Tesis para optar el grado académico de magíster en problemas de aprendizaje. Universidad César Vallejo, Lima, Perú.

Arbuckle, J. (2017). Amos (Versión 25.0) [Programa Informático]. Chicago: IBM SPSS.

Arnau, J. (1995). Metodología de la investigación psicológica. En M. T. Anguera, J. Arnau, M. Ato, R. Martínez-Arias, J. Pascual y G. Vallejo (Eds.), Métodos de investigación en psicología (pp. 23-44). Madrid: Síntesis. 
Bentler, P. (1990). Comparative fit índices in structural models. Psychological Bulletin, 107(2), 238-246.

Bolívar, D. P., Ballesteros, L. P., \& Ramírez, C. S. (2014). Relación entre procrastinación académica y ansiedad-rasgo en estudiantes de psicología. Cuadernos hispanoamericanos de Psicología, 14(1), 31-44.

Buela-Casal, G., Guillén-Riquelme, A., \& Seisdedos Cubero, N. (2015). Cuestionario de ansiedad estado-rasgo: Adaptación española. Madrid: TEA Ediciones.

Bustos, V., Oliver, A., \& Galiana, L. (2015). Validación del Autoconcepto Forma 5 en Universitarios Peruanos: Una herramienta para la psicología positiva. Psicologia: Reflexão e Crítica, 28(4), 690-697. Disponible en: http://www.redalyc.org/articulo. oa?id=18842573007

Caballo, V. E., Piqueras, J. A., Antona, C., Irurtia, M. J., Salazar, I. C., Bas, P., \& Salavera, C. (2018). La autoestima y su relación con la ansiedad social y las habilidades sociales. Psicología Conductual, 26(1), 23-53. Disponible en: https://zaguan.unizar. es/record/70886/files/texto_completo.pdf

Cárdenas, M. T. C., Castillo, C. A. G., \& Camargo, Y. S. (2011). Relación entre niveles de ansiedad y estrategias de afrontamiento en practicantes de psicología de una universidad colombiana. International Journal of Psychological Research, 4(1), 50 57. Disponible en: http://www.redalyc.org/articulo.oa?id=299022819007

Cardona-Arias, J. A., Perez-Restrepo, D., Rivera-Ocampo, S., Gómez-Martínez, J., \& Reyes, Á. (2015). Prevalencia de ansiedad en estudiantes universitarios. Diversitas: Perspectivas en Psicología, 11(1), 79-89. Disponible en: http://www.redalyc.org/ articulo.oa? $\mathrm{id}=67943296005$

Cohen, J. (1988). Statistical Power Analysis for the Behavioral Sciences. $2^{\text {nd }}$ ed. New York: Lawrence Erlbaum Associates.

Dominguez-Lara, S. A., Bonifacio-Vilela, M., \& Caro-Salazar, A. (2016). Prevalencia de ansiedad ante exámenes en una muestra de estudiantes universitarios de Lima Metropolitana. Revista de Psicología, 6(2), 45-56. Disponible en: http://35.161.26.73/ index.php/psicologia/article/view/155

Dominguez-Lara, S. \& Rodriguez, A. (2017). Índices estadítiscos de modelos bifactor. Interacciones, 3(2), 59-65. doi:10.24016/2017.v3n2.51

Emmelkamp, P. M., \& Ehring, T. (Eds.). (2014). The Wiley handbook of anxiety disorders. John Wiley \& Sons Incorporated.

Esnaola, I., Goñi, A., \& Madariaga, J. M. (2008). El autoconcepto: perspectivas de investigación. Revista de psicodidáctica, 13(1), 69-96. Disponible en: http://www. redalyc.org/articulo.oa?id=17513105

Esnaola, I., Rodríguez, A., \& Goñi, E. (2011). Propiedades psicométricas del cuestionario de Autoconcepto AF5. Anales de Psicología/Annals of Psychology, 27(1), 109-117. https://revistas.um.es/analesps/article/view/113531/107521

Eysenck, M. (2014). Anxiety and cognition: A unified theory. New York: Psychology Press. Recuperado de: https://books.google.com.pe/books?id=QzelAgAAQBAJ\&p 
$\mathrm{g}=\mathrm{PT} 225 \& \mathrm{dq}=$ the + psychology + of + anxiety\&hl=es\&sa=X\&ved=0ahUKEwiSz_rh0 bDlAhVSzlkKHUJcAT8Q6AEIUjAF\#v=onepage\&q\&f=false

Flores-Kanter, P. E., Dominguez-Lara, S., Trógolo, M. A., Medrano, L. A. (2018). Best practices in the use of bifactor models: Conceptual grounds, fit indices and complementary indicators. Revista Evaluar, 18(3), 44-48. Retrieved from https:// revistas.unc.edu.ar/index.php/revaluar

Fuentes, M. C., García, J. F., Gracia, E., \& Lila, M. (2011). Autoconcepto y ajuste psicosocial en la adolescencia. Psicothema, 23(1), 7-12. Disponible en: http://www. redalyc.org/articulo.oa?id=72717207002

García, A. G. G., \& Velázquez, M. G. L. (2018). Autoeficacia académica y ansiedad, como incidente crítico, en mujeres y hombres universitarios. Revista Costarricense de Psicología, 37(1), 1-25. DOI: http://dx.doi.org/10.22544/rcps.v37i01.01

García, B. (2001). Cuestionario de autoconcepto Garley CAG. Madrid, España: Instituto de Orientación Psicológica EOS, Madrid, España.

García, F. \& Musitu, G. (2001). Autoconcepto Forma 5. AF5. Manual. Madrid: TEA.

García, F. \& Musitu, G. (2014). Autoconcepto Forma 5. AF5. Manual (Cuarta edición, revisada y ampliada). Madrid: TEA.

González, M. T., Landero, R., \& García-Campayo, J. (2009). Relación entre la depresión, la ansiedad y los síntomas psicosomáticos en una muestra de estudiantes universitarios del norte de México. Revista panamericana de salud pública, 25, 141-145. Disponible en https://www.scielosp.org/article/rpsp/2009.v25n2/141-145/es/

Hernández, R., Fernández, C. \& Baptista, M. (2014). Metodología de la investigación (6ta ed.). México: Mc Graw Hill / Interamericana.

Light, R. J., Singer, J. D., \& Willett, J. B. (1990). By design: Planning research on higher education. Cambridge, MA: Harvard University Press.

Londoño, D. M. M., Lubert, C. D., Sepúlveda, V. E. P., \& Ferreras, A. P. (2019). Estandarización de la Escala de autoconcepto AF5 en estudiantes universitarios colombianos. Ansiedad y Estrés. https://doi.org/10.1016/j.anyes.2019.06.001

López, B. G., Esteban, P. R. G., Peris, F. S., Ros, C. R., \& Carbonell, B. S. (2009). La influencia del autoconcepto en el rendimiento académico en estudiantes universitarios. Revista Española de Orientación y Psicopedagogía, 20(1), 16-28. Disponible en: http://www.redalyc.org/articulo.oa?id=338230781003

MacCallum, R.C., Browne, M.W. \& Sugawara, H.M. (1996). Power Analysis and determination of sample size for covariance structure modeling of fit involving particular measure of model. Psychological Methods, 13(2), 130-149.

Matalinares, M., Arenas, C., Yaringaño, J., Sotelo, L., Sotelo, N., Díaz, G., ... \& Pezua, M. (2011). Factores personales de resiliencia y autoconcepto en estudiantes de primaria de Lima metropolitana. Revista de investigación en psicología, 14(1), 187-208. Disponible en: https://revistasinvestigacion.unmsm.edu.pe/index.php/psico/article/ view/2083 
Massenzana, F. B. (2017). Autoconcepto y autoestima: ¿sinónimos o constructos complementarios? \{PSOCIAL\}, 3(1), 39-52. Disponible en: https://publicaciones. sociales.uba.ar/index.php/psicologiasocial/article/view/2336/1984

Muñiz, J., Elosua, P., \& Hambleton, R. K. (2013). Directrices para la traducción y adaptación de los tests: segunda edición. Psicothema, 25(2), 151-157.). Directrices para la traducción y adaptación de los tests: segunda edición. Psicothema, 25(2), 151-157. Doi: 10.7334/psicothema2013.24

Nunes, M., Rigotto, D. M., Ferrari, H. \& Marín, F. J. (2012). Soporte social, familiar y autoconcepto: relación entre los constructos. Psicología desde el Caribe, 29(1), 1-18. Disponible en: http://www.redalyc.org/articulo.oa?id=21323171002

Núñez, I. y Crismán, R. (2016). La ansiedad como variable predictora de la autoestima en adolescentes y su influencia en el proceso educativo y en la comunicación. Revista Iberoamericana de Educación, 71(2), 109-128. Disponible en: https://dialnet.unirioja. es/servlet/articulo?codigo $=5820976$

Ospina-Ospina, F. D. C., Hinestrosa-Upegui, M. F., Paredes, M. C., Guzmán, Y., \& Granados, C. (2011). Síntomas de ansiedad y depresión en adolescentes escolarizados de 10 a 17 años en Chía, Colombia. Revista de salud pública, 13, 908-920. Disponible en: https:/www.scielosp.org/scielo.php?pid=S0124-00642011000600004\&script=sci abstract

Quiroz, C. O. A., \& Flores, R. G. (2007). Ansiedad y depresión en adultos mayores. Psicología y salud, 17(2), 291-300. https://doi.org/10.25009/pys.v17i2.714

Rodrich, P. A. (2019). Autoestima y ansiedad estado-rasgo en jóvenes universitarios de la ciudad de Lima. Tesis para optar el título profesional de licenciada en psicología. Universidad de Lima, Lima, Perú.

Rodríguez, U., Amaya, A.M., \& Argota, A.P. (2011). Inteligencia emocional y ansiedad en estudiantes universitarios. Psicogente, 14(26), 310-320. Disponible en: https://www. redalyc.org/articulo.oa?id=4975/497552359007

Sánchez, H. \& Reyes, C. (2015). Metodología y diseños en la investigación científica. 5ta ed. Lima, Perú: Business Suport Aneth.

Sánchez, M. L. Z., López, R. C., Moral, P. V., \& Zagalaz, J. C. (2017). Relación entre autoconcepto físico, ansiedad y personalidad manifestada en usuarios de gimnasios. RETOS. Nuevas Tendencias en Educación Física, Deporte y Recreación, 32, 53-57. Disponible en: http://www.redalyc.org/articulo.oa?id=345751100011

Sandín, B., Chorot, P., Santed, M. A., \& Valiente, R. M. (2002). Análisis factorial confirmatorio del Índice de Sensibilidad a la Ansiedad para Niños. Psicothema, 14(2), 333-339. Disponible en: http://www.redalyc.org/articulo.oa?id=72714222

Shavelson, R. J., Hubner, J. J., \& Stanton, G. C. (1976). Self-Concept: Validation of Construct Interpretations. Review of Educational Research, 46(3), 407-441. doi:10.3102/00346543046003407

Spielberger, C. D. (1966). Theory and research on anxiety. Academic Press: New York and London. 
Spielberger, C. D., Gorsuch, R. L. y Lushene, R. E. (2008). STAI. Cuestionario de ansiedad estado-rasgo ( $7^{\mathrm{a}}$ ed. rev.). Madrid: TEA

Spielberger, C. D., Gorsuch, R. L. y Lushene, R. E. (2015). STAI. Cuestionario de ansiedad estado-rasgo ( $9^{\mathrm{a}}$ ed. rev.). Madrid: TEA

Vélez, D. M. A., Garzón, C. P. C., \& Ortíz, D. L. S. (2008). Características de ansiedad y depresión en estudiantes universitarios. International Journal of Psychological Research, 1(1), 34-39. Disponible en http://www.redalyc.org/articulo. oa?id=299023503006 
